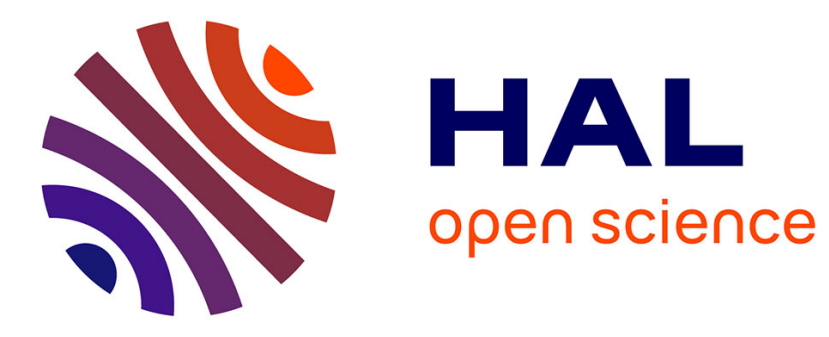

\title{
Amplitude-based data selection for optimal retrospective reconstruction in micro-SPECT
}

Marine Breuilly, Grégoire Malandain, Julien Guglielmi, Robert Marsault, Thierry Pourcher, Philippe R. Franken, Jacques Darcourt

\section{To cite this version:}

Marine Breuilly, Grégoire Malandain, Julien Guglielmi, Robert Marsault, Thierry Pourcher, et al.. Amplitude-based data selection for optimal retrospective reconstruction in micro-SPECT. Physics in Medicine and Biology, 2013, 58 (8), pp.2657-2674. 10.1088/0031-9155/58/8/2657 . hal-00799381

\section{HAL Id: hal-00799381 \\ https://hal.inria.fr/hal-00799381}

Submitted on 12 Mar 2013

HAL is a multi-disciplinary open access archive for the deposit and dissemination of scientific research documents, whether they are published or not. The documents may come from teaching and research institutions in France or abroad, or from public or private research centers.
L'archive ouverte pluridisciplinaire HAL, est destinée au dépôt et à la diffusion de documents scientifiques de niveau recherche, publiés ou non, émanant des établissements d'enseignement et de recherche français ou étrangers, des laboratoires publics ou privés. 


\title{
Amplitude-based data selection for optimal retrospective reconstruction in micro-SPECT
}

\author{
M Breuilly ${ }^{1,2}$, G Malandain ${ }^{1}$, J Guglielmi $^{2}$, R Marsault ${ }^{2}, \mathbf{T}$ \\ Pourcher $^{2}$, PR Franken ${ }^{2}$ and J Darcourt ${ }^{2}$ \\ ${ }^{1}$ INRIA Sophia Antipolis - Méditerranée, Sophia Antipolis, France \\ ${ }^{2}$ Laboratoire TIRO, Commissariat à l'Énergie Atomique et aux Énergies \\ Alternatives (CEA) / Université de Nice Sophia Antipolis / Centre Antoine \\ Lacassagne, Nice, France \\ E-mail: Marine.Breuilly@inria.fr
}

\begin{abstract}
Respiratory motion can blur the tomographic reconstruction of PET or SPECT images, which subsequently impair quantitative measurements, e.g. in the upper abdomen area. Respiratory signal phase-based gated reconstruction addresses this problem, but deteriorates the signal-to-noise ratio and other intensity-based quality measures. This article proposes a $3 \mathrm{D}$ reconstruction method dedicated to micro-SPECT imaging of mice. From a 4D acquisition, the phase images exhibiting motion are identified and the associated list-mode data are discarded, which enables the reconstruction of a 3D image without respiratory artefacts.

The proposed method allows a motion-free reconstruction exhibiting both satisfactory count statistics and accuracy of measures. With respect to standard 3D reconstruction (NG3D) without breathing motion correction, an increase of $14.6 \%$ of the mean SUV has been observed, while, with respect to a gated $4 \mathrm{D}$ reconstruction (G4D), up to $60 \%$ less noise and an increase of up to $124 \%$ of the SNR have been demonstrated.
\end{abstract}

SPECT, respiratory motion, image-based motion detection, amplitude-based gating and rodents

Submitted to: Phys. Med. Biol. 


\section{Introduction}

Molecular pre-clinical imaging is a major research tool which provides non-invasive in vivo information on cellular processes and allows longitudinal studies (Pysz et al. 2010, Franc et al. 2008, Kang \& Chung 2008). In oncology, obtaining measurements of tumour characteristics is mandatory. Emission tomography (ET) quantification is hampered by physically induced biases such as attenuation, scatter and partial volume effect, as well as by motion artefacts. In clinical imaging, the physical biases can be corrected in Positron Emission Tomography (PET) as well as in Single-Photon Emission Computed Tomography (SPECT) (Ritt et al. 2011). However, there are few studies addressing specifically the quantification issues in pre-clinical imaging. Concerning physical biases, Hwang et al. (Hwang et al. 2008) showed that for technetium-99m ${ }^{99 \mathrm{~m}} \mathrm{Tc}$ ), attenuation and scatter errors are reduced in small animal SPECT compared to clinical SPECT. Therefore, respiratory motion compensation is one of the main obstacles of accurate quantification in micro-SPECT ( $\mu$-SPECT).

Computed Tomography (CT), PET or SPECT images are produced by a tomographic reconstruction from the projections of the object of interest, with the implicit assumption that the imaged object remains still during the projection acquisition. Motivated by the respiratory and cardiac motion observed in clinical practice, a number of motion handling methods have been developed, with the goal of reconstructing images free of motion artefacts. A trivial approach consists in controlling the considered motion during the acquisition, by breath-holding, for example. This is only valid for human imaging and short-time acquisition techniques such as CT or Magnetic Resonance (MR).

Motion artefacts can also be handled by gating protocols. An additional signal, which is considered representative of the motion of interest, is recorded and synchronised with the data acquisition. Reconstruction is then based on a selection of the acquired data. Such signals can be acquired by an external device and include electrocardiogram (Livieratos et al. 2006), Real-time Position Management (Grotus et al. 2009) or Multidimensional Respiratory Gating (Nehmeh et al. 2011), pressure sensor (chest belt) (Chang et al. 2010, van Elmpt et al. 2011), and optical fiber (Burk et al. 2012). These signals can also be extracted directly from the data itself, by looking for time variation in raw projections in CT (Kyriakou \& McKenzie 2011) or in ET (Bundschuh et al. 2008, Schleyer et al. 2009).

For periodic signals, phase-based gating consists in dividing the period into several phases of equal duration, with the assumption that the motion can be neglected within each phase. This method is used for both cardiac (Büther et al. 2010) and respiratory (Bettinardi et al. 2010, Bundschuh et al. 2008, Grotus et al. 2009, Livieratos et al. 2006) gating for human imaging and allows the reconstruction of dynamic or $4 \mathrm{D}(3 \mathrm{D}+\mathrm{t})$ images. Using only a fraction of the acquired data deteriorates the signal-to-noise ratio (SNR) of each 3D image. Therefore, Dawood et al. propose to co-register each 3D image and then to sum them up (Dawood et al. 2006). With this method, obtained 3D 
images have minimal motion artefacts and improved SNR.

Furthermore, amplitude-based gating assumes that the signal amplitude is representative of the motion of interest. A range of amplitude values is selected, and the associated acquired data are used for reconstruction. For instance, images at end of exhalation or inhalation can be reconstructed by selecting either the lowest or the highest amplitude values. In human imaging, such a method has been used for cardiac motion in SPECT (Kovalski et al. 2007) and for respiratory motion in PET (Bettinardi et al. 2010, Chang et al. 2010, Nehmeh et al. 2011, Schleyer et al. 2009, van Elmpt et al. 2011).

When a motion model is available, some authors proposed to incorporate it into the tomographic reconstruction procedure in order to take into account all the acquired data to reconstruct a 3D still image. In human imaging, cardiac (Blondel et al. 2004) and respiratory (Lamare et al. 2007, Reyes et al. 2007) motion models have been developed. In addition to the difficulty in defining an accurate motion model, such methods also imply a huge computational cost.

In human imaging, motion compensation has been studied in CT or PET (Nehmeh \& Erdi 2008, Lucignani 2009). In pre-clinical imaging, the methods are similar. For instance, Kuntz et al. used phase-based gating (Kuntz et al. 2010). However the literature is less abundant than in the clinical field and mostly concerns $\mu$-CT (Guo et al. 2011, Martiniova et al. 2010).

In this article, we propose an amplitude-based gating reconstruction method in mice $\mu$-SPECT. The signal obtained from an external pressure sensor is filtered and allows the reconstruction of an intial 4D image, which is then analysed to detect the respiratory induced motion. We take advantage of the particular breathing pattern of anaesthetized mice to detect motion-corrupted phases, and subsequently use all raw data corresponding to the motionless phases to reconstruct a single motionless 3D image.

\section{Materials and methods}

\subsection{Animal model}

The different methods were tested on data obtained on 3 female mice of a mouse model. The mouse model consisted of NOD-SCID mice with intra-peritoneally injection of 1.5 million cells of rat colonic adenocarcinoma expressing the Natrium Iodide Symporter (PROb-mNIS) in $50 \mu \mathrm{L}$ of Phosphate Buffered Saline. SPECT/CT acquisitions were performed after 1, 2, and 3 weeks of growth, and PROb-mNIS peritoneal nodules of NIS-transfected cells were observed. However, we considered only 8 acquisitions out of the 9 for our study, since the Biovet pressure signal failed for one acquisition.

Animal housing and procedures were conducted according to the guidelines of the French Agriculture Ministry and were approved by the local ethics committee. 


\subsection{Imaging protocol}

We acquired experimental animal images using a dedicated small animal SPECT/CT scanner (eXplore speCZT CT 120, GE Healthcare Bioscience, London ON, CA).

The SPECT imaging part of this camera consists of a fixed full-ring of detectors coupled to a rotating 7-pinhole collimator for mice. The ring of detectors is composed of 10 panels of 4 CZT detectors. SPECT acquisitions lasted around $18 \mathrm{~min}$. The output of the camera is a list-mode record of all CZT detected events : each event consists of the detection time (ms), the gating indices, and the properties of the detected photon. SPECT reconstructions used data from the list-mode in the energy window from 125 to $150 \mathrm{keV}$, according to the energy of $99 \mathrm{mTc}$.

Mice were injected intra-peritoneally $180 \mathrm{MBq}$ in $400 \mu \mathrm{L}$ of ${ }^{99 \mathrm{~m}} \mathrm{Tc}$-pertechnetate. Five minutes later, the animals were anaesthetized with isoflurane $(1.3 \% \mathrm{v} / \mathrm{v})$ (Baxter, France). The animals were placed prone and freely breathing (without mechanical ventilation) on the bed and kept sedated during the whole imaging protocol, using inhaled anaesthetic.

A monitoring system (BioVet, m2m Imaging Corporation, Newark, USA) was used with a pneumatic pressure sensor. The pressure sensor was placed under the animal abdomen. The pressure signal was recorded (1 ms-sampled rate) and used to monitor the animal anaesthesia.

Body temperature was maintained at $37^{\circ}$ Celsius and anaesthetized gas rate was regularly controlled to keep the respiratory rate between 60 and 100 breaths per minute. Once a stabilized respiratory rate was reached, the SPECT acquisition started.

At the beginning of each acquisition, an amplitude threshold was arbitrarily fixed in order to detect the falling edge of the pressure signal variation. Each time the signal passed this threshold, a trigger was recorded in the list-mode SPECT acquisition data to be used for reconstruction. We assumed that this trigger corresponded to the same time in the cycle for each respiratory cycle. A respiratory cycle is then defined by the interval between two consecutive triggers.

\subsection{Reconstruction algorithm}

SPECT reconstruction was done using the Ordered Subset Expectation Maximization (OSEM) algorithm (Hudson \& Larkin 1994). We used from 7 to 9 subsets and from 6 to 9 iterations, the number of EM equivalent updates ranged from 54 to 56 . For a given set of data, the number of subsets and iterations were kept constant for all the reconstruction methods.

\section{4. "Standard" reconstruction methods}

In this section, we describe the different kinds of SPECT reconstruction methods for the list-mode, resulting in either $3 \mathrm{D}$ or $4 \mathrm{D}$ images. We start with the reconstruction 
that are provided by the camera and then we introduce the improvements which lead to our final reconstruction scheme: a breath-hold like 3D image.

2.4.1. Non-gated 3D reconstruction Non-gated 3D reconstruction (NG3D) consists in reconstructing a single 3D image using all data recorded in the list-mode within the range of energy corresponding to the radiopharmaceutical used.

2.4.2. Gated 4D reconstruction Gated 4D reconstruction (G4D) consists in reconstructing a temporal sequence of $N$ 3D images. The number of phases $N$ is set to 15 in the experiments. Using the trigger set in pressure signal, we compute the respiratory cycle average duration, denoted by $\bar{C}$, over the whole acquisition. The duration of each reconstruction window is then $D=\bar{C} / N$. Therefore the $n^{\text {th }}$ gating window of the $i^{\text {th }}$ cycle is defined by the temporal interval $\left[t_{i}+(n-1) D, t_{i}+n D\right.$ [ where $t_{i}$ is the time of the $i^{\text {th }}$ trigger and $n \in[1, N]$. A 3D image for each gating window is then reconstructed from the data of the corresponding window. The raw data (list-mode) used is the same as for NG3D reconstruction.

\subsection{Proposed Method}

The proposed method is a combination of three main steps that are described here: a cycle filtering which consists in cycle pre-selection according to duration, a cycle resampling, and an image-based motionless phase detection. This method denoted by breath-hold like 3D reconstruction (BH3D) gives 3D images without motion artefact.

2.5.1. Cycle selection As the acquisitions are done on free-breathing mice, the respiratory rate is linked to the level of isoflurane. Even once the steady state of narcosis is reached, some extreme cycles lengths are observed. These outlier cycles may impair the reconstruction by inducing a non coherent motion in images: moving structures appear blurred. Consequently, we discarded the outlier cycles from the list-mode. The study of the pressure signal gives the distribution of respiratory cycle duration and allows the identification of the outlier cycles. A new list-mode data set is generated where the corresponding outlier cycles are discarded. We experimentally set the acceptance cycle duration window to the mean cycle duration $+/-3$ standard deviations $[\bar{C}-3 \sigma ; \bar{C}+3 \sigma]$.

Figure 1 presents a typical example of the distribution of cycle durations for an acquisition. The acceptance set to $[\bar{C}-3 \sigma ; \bar{C}+3 \sigma]$ will guarantee to keep around $99.7 \%$ of the cycle if the distribution is Gaussian, while it will discard the outlier cycles.

After rejection of the outlier cycles, a new respiratory cycle average duration $\bar{C}_{s}$ is

calculated and leads to a new duration of gating window $D_{s}=\bar{C}_{s} / N$. The $n^{\text {th }}$ gating window of the $i^{\text {th }}$ cycle is defined by $\left[t_{i}+(n-1) D_{s}, t_{i}+n D_{s}\right.$ [ where $t_{i}$ is the time of the $i^{\text {th }}$ trigger. 
2.5.2. Cycle resampling Obviously, even after the cycle selection step, the remaining respiratory cycles still exhibit some length variations.

About half of the cycles are longer than $\bar{C}_{s}$ and their trigger time $t_{j}$ satisfies $t_{j+1}-t_{j}>\bar{C}_{s}$. As a consequence, the counts after the last gating window of this cycle and before the next trigger signal (i.e. in the interval $\left[t_{j}+\bar{C}_{s}, t_{j+1}[\right.$ ) will not be used at all in the reconstruction process. This is illustrated by figure $2(\mathrm{~b})$.

Conversely, the other half of the cycles are shorter than the average cycle duration $\bar{C}_{s}$ and their trigger time $t_{k}$ satisfies $t_{k+1}-t_{k}<\bar{C}_{s}$. As a consequence, the counts of the last gating windows defined for this cycle (in the interval $\left[t_{k+1}, t_{k}+\bar{C}_{s}\right.$ [) also belong to the first gating windows of the next cycle, meaning that those counts will be used twice in the reconstruction process for different phase images. This is illustrated by figure $2(\mathrm{a})$.

These observations motivate a temporal resampling of the counts so that the interval between two trigger signals is equal to the cycle average duration $\bar{C}_{s}$. This ensures that each event will be used once and only once in the reconstruction process. Therefore the detection time $t$ of an event located between the trigger signals $t_{i}$ and $t_{i+1}$ will be changed to

$$
t^{\prime}=(i-1) \bar{C}_{s}+\left(t-t_{i}\right) \frac{\bar{C}_{s}}{t_{i+1}-t_{i}}
$$

while the trigger signals become $t_{i}^{\prime}=(i-1) \bar{C}_{s}$. This temporal normalisation results in a new list-mode data set that enables the reconstruction of a gated $4 \mathrm{D}$ image, denoted G4DSR.

2.5.3. Image-based motionless phase detection Anaesthetised mice exhibited a particular breathing rhythm characterized by long intervals (around $2 / 3$ of the cycle) without respiratory motion separated by gasping breaths, as mentioned in (Cavanaugh et al. 2004) and exemplified in the pressure signal in figure 3(a). Extracting the list-mode data corresponding to the rest intervals will allow then to reconstruct a breath-hold like image. However, the visual inspection of the 4D images (either G4D or G4DSR) reveals that the observed motion is temporally shifted with respect to the observed variations of the respiratory signal. Therefore, the latter can not be used directly for motion detection and motivates the design of a dedicated image-based motion detection method from a $4 \mathrm{D}$ sequence with 15 phases (here the G4DSR images, i.e. the 4D image after cycle selection and temporal resampling).

This method is based on the observation that, around the borders of high contrasted areas, the time-course uptake value of a given voxel may exhibit a large variation due to spatial displacement. It consists in the study of time-course uptake values of voxels in the abdominal region - assuming that a spatial position in an image should always refer to the same object along time if there is no motion.

The workflow for detecting motionless phases can be described as followed:

(i) Intensity normalisation of the $3 \mathrm{D}$ images $I_{n}, n \in[1, N]$ resulting from the $4 \mathrm{D}$ 
reconstruction according to the proportion of available counts that has been used for reconstruction.

$$
\forall x: \hat{I}_{n}(x)=I_{n}(x) \frac{\text { total number of counts }}{\text { number of counts used to reconstruct } I_{n}}
$$

(ii) Spatial regularization with a 3D Gaussian filter $(\sigma=1.00)$ of the normalised images $\hat{I}_{n}$ yielding $\tilde{I}_{n}$.

(iii) Volume of interest definition. Since we are interested in abdominal lesions, we restrict the above analysis to a sub-volume containing only the abdominal area.

(iv) Generation of an amplitude image. A 3D image of intensity variation is generated from the images $\tilde{I}_{n}$. For a given voxel $x$, the uptake amplitude can be simply computed by subtracting the minimal uptake value from the maximal one, resulting in an amplitude image $A$.

$$
\forall x: A(x)=\max \left\{\tilde{I}_{n}(x)\right\}_{n \in[1, N]}-\min \left\{\tilde{I}_{n}(x)\right\}_{n \in[1, N]}
$$

(v) Generation of a mask $M$ of regions of potential motion. This mask aims at isolating voxels with large variations of time-course uptake values. This mask is obtained by thresholding the amplitude image $A$ at $15 \%$ of its maximum value, yielding segmented regions with potential spatial displacement.

(vi) Detection of the gasping breaths. We observe that around $2 / 3$ of the respiratory cycle do not exhibit significant motion. Since $N$ phases are reconstructed, with $N=$ 15, this correspond to around 10 phases without motion while the motion-induced large uptake variations are then mostly due to the other 5 phases. Therefore, for each voxel $x$, we compute the amplitude after discarding every possible sequence of 5 consecutive phases. We define $S_{m}$ as a sequence of 5 consecutive phases:

$$
S_{m}=\{k \in[1, N] \mid \exists j \in[0,4]: k-m \equiv j[N]\}
$$

The discarded sequence yielding the smallest amplitude is associated to a possible motion under the considered voxel. This sequence is defined by its first phase $\hat{m}(x)$ computed by:

$$
\hat{m}(x)=\arg \min _{m \in[1, N]}\left(\max \left\{\tilde{I}_{n}(x)\right\}_{n \in[1, N] \backslash S_{m}}-\min \left\{\tilde{I}_{n}(x)\right\}_{n \in[1, N] \backslash S_{m}}\right)
$$

We cumulate the obtained sequences $S_{\hat{m}(x)}$ into a histogram $h$.

$$
\forall n \in[1, N], h(n)=\operatorname{card}\left\{x \in M / n \in S_{\hat{m}(x)}\right\}
$$

The resulting histogram is automatically thresholded using the Otsu method (Otsu 1975), yielding two groups of phases. The smallest group (typ. 5 to 6 phases) is associated with motion state and the largest group (typ. 10 or 9 phases) is associated with the motionless state. The first phase of the latter group is denoted by $\hat{p}$ and the number of phase is denoted $L$. The results of the histogram thresholding (table 1) confirms the visual inspection illustrated by figure 3(b) : the phases associated with the motion state are shifted with respect to the pressure signal peak. 
2.5.4. Breath-hold like 3D reconstruction (BH3D) Breath-hold like 3D reconstruction consists in reconstructing a single 3D image using all data belonging to motionless phases. More precisely this reconstruction method considers counts that lie in intervals of length $L D_{s}$ beginning at phase $\hat{p}$, i.e. $\left[t_{i}^{\prime}+(\hat{p}-1) D_{s}, t_{i}^{\prime}+(\hat{p}-1) D_{s}+L D_{s}\right.$ [ in the list-mode data set (after the temporal resampling).

\subsection{Image value normalisation}

Reconstructed images were converted into Standardized Uptake Values (SUV) for comparison purpose. The conversion is divided in two steps.

First, all the reconstructed images (3D images, as BH3D, or 3D phase images from 4D images, as G4D, G4DSR) were normalised to NG3D. The values of the reconstructed voxels depend directly on the number of counts used in the reconstruction process. NG3D is the 3D image reconstructed with all the available counts, while all other images only uses a fraction of them. Let us denote by $N c(I)$ the number of counts used for the reconstruction of image $I$. By mutiplying the values of image $I$ after reconstruction by the ratio $N c(N G 3 D) / N c(I)$, the resulting values are comparable with the ones of NG3D.

Second, the values are converted into Standardized Uptake Values (SUV). Thanks to the previous normalisation, values of all reconstructed images are comparable and the conversion into SUV is the same for all images. Image values are first converted into uptake values by calibration using an external known 99mTc source acquired simultaneously with the image. SUV values are obtained by normalising the uptake values by the total injected activity divided by the animal weight.

\subsection{Image measurements}

A number of measures, either image-based or lesion-based, were performed for each reconstructed image.

A volume of interest was manually delineated in the liver with the help of both anatomical (CT) and SPECT (NG3D) images avoiding any abnormal lesion, yielding a volume of homogeneous SUV. Calculating the standard deviation (SD) over this region allow the noise of the reconstructed image to be estimated.

Peritoneal metastases were localized by three experts with the help of maximum of intensity projections (MIP), and a VOI was delineated around every identified lesion. The maximal SUV value, $\mathrm{SUV}_{\max }$, is calculated, but was not directly used for comparison purposes since it is sensitive to noise, especially for small lesions. The following measurements are then made within every VOI:

- $\mathrm{SUV}_{\text {peak }}$ : the mean SUV of the $5 \%$ of highest values;

- $\mathrm{SUV}_{\text {mean }}$ : the mean $\mathrm{SUV}$ of values over $40 \%$ of $\mathrm{SUV}_{\max }$;

- lesion volume: volume at $40 \%$ of the $\mathrm{SUV}_{\max }$ (given in $\mathrm{mm}^{3}$ );

- lesion SNR: ratio of lesion $\mathrm{SUV}_{\text {mean }}$ to the liver VOI SD. 
VOI were drawn using AMIDE software (Loening \& Gambhir 2003). No correction for partial volume effect were performed.

\subsection{Statistical tools}

Paired variables were compared using t-tests (Matlab R2009b; Mathworks, Natick, MA). Image-based measures (i.e. noise), which can be considered as intrinsic, were compared using paired t-tests. Lesion-based measures ( $\mathrm{SNR}, \mathrm{SUV}_{\text {peak }}, \mathrm{SUV}_{\text {mean }}$, and volume) were not directly comparable since they vary from lesion to lesion. Therefore they were compared using ratio paired t-tests that correspond to paired t-tests on their logarithms (base 10).

\section{Results}

\subsection{Lesions distribution}

Figure 4 presents the volume rendering of fused CT and SPECT (NG3D) images of peritoneal carcinoma lesions after 3 weeks of growth. Figure 4 illustrates both biological and imaging challenges as there are numerous small peritoneal carcinoma lesions, distributed throughout the abdomen.

A total of 76 foci of ${ }^{99 m}$ Tc-uptake were detected on the 8 acquisitions (3 to 14 lesions per acquisition). They were located in the abdomen, showing NIS-expressing tumour tissue, and corresponded to peritoneal carcinoma nodules. These nodules were small, their size (equivalent spherical diameter) ranged from 1.09 to $2.34 \mathrm{~mm}$ (average $1.55 \pm 0.28 \mathrm{~mm})$. Twenty-seven lesions were located in the upper abdominal area (above the stomach), 31 were located in the middle abdominal area (at stomach level), and 18 were located in the lower abdominal area (below the stomach).

\subsection{Respiratory signal analysis}

Figure 3 (a) presents a sample of pressure signal of free-breathing mice. Table 1 presents the results of the pressure signal analysis on the 8 acquisitions. The average duration of the respiratory cycle varies from $658 \mathrm{~ms}$ to $766 \mathrm{~ms}$ while its standard deviation ranges from 44 to $153 \mathrm{~ms}$.

This signal is characterised by a still phase at the end of exhalation. The imagebased motionless phase detection demonstrated that the shorter phase associated with motion state lasts 5 or 6 phases out of 15 (see table 1) which corroborates our initial observation of a duration of around $1 / 3$ of the respiratory cycle. Moreover, it has been detected that the motion state is time-shifted away from the pressure signal. According to the image-based motionless phase detection, the time shift varies from 3 to 5 phases.

Figure 3 (b) presents the normalised average respiratory cycle (continuous curve) for one acquisition, after the cycle selection and the cycle resampling steps, with the result of the image-based motionless phase detection (dashed curve). A horizontal line 
represents the threshold computed by the Otsu method. This threshold separates the phases in two groups, the ones above the threshold are associated with the motion phase, the others (below the threshold) are associated with the motionless phase.

We did further experiments on the observed time shift between the imagebased detected motion and the pressure signal. For this purpose, one mouse has undergone several 4D SPECT acquisitions (with different gating triggers) and one 4D CT acquisition. The proposed image-based method has been used on all the 4D SPECT acquisitions and on the 4D CT acquisition. Changing the trigger parameters on the pressure signal (rising/falling edge, with/without delay) for the consecutive SPECT acquisitions of the same animal did not change the location of the peak (data not presented here). The peak of the motion detected in the 4D CT appeared at the same location as in SPECT, with a temporal shift with respect to the pressure signal (figure 5). Last, we computed the lung volume from the CT images along the phases with an adhoc and simple method (Gaussian smoothing, threshold between -650 HU and -250 HU, and morphological closing). Again, the peak of volume change appeared at the same location. There is a discontinuity in the lung volume variations (at phase \#10): the corresponding reconstructed CT image appears to exhibit different characteristics which explained the lung segmentation differences.

\subsection{Sensitivity of the image-based motion detection with respect to noise}

We investigated the ability of the proposed method to detect the motion in noisy images. To that end, we extracted from one 4D SPECT sequence a series of 3D images containing a moving lesion. This lesion has been thresholded, and different (white and gaussian) noise levels have been added to the binary images. It appears from our experiments that the image-based motion detection method is always successful in detecting the motion phases for SNRs above 2.5.

\subsection{Image statistics}

The number of counts used for each reconstruction method were compared to the total number of acquired counts. Table 2 presents the figures concerning the average percentages on the set of 8 acquisitions. By definition, the 3D reconstruction method NG3D used $100 \%$ of the counts.

$153 \mathrm{D}$ phase images were reconstructed by the G4D method. The average percentage of counts used for the reconstruction of each 3D phase image is then around $1 / 15$ th of the counts (6.65\%). However, because of the variation of respiratory cycle duration, $3.64 \%$ of the counts are not taken in account while $3.51 \%$ of them are used twice (i.e. for the reconstruction of two different phases). If the total number of used counts is defined as the sum of the number of counts used for each phase reconstruction, it appears that this total number may exceed $100 \%$ if the number of counts used twice is larger than the number of omitted counts. As a consequence, the average total of counts used for the reconstruction of the 15 phases is $99.76 \%$ with a standard deviation 
of $2.88 \%$. The twice used counts were not evenly distributed among phases, and are more likely to occur in the first phases: an average of $31.44 \%$ (resp. 9.67\%) of counts used for phase 1 (resp. phase 2) were already used in a preceding phase. Twice used counts appeared up to the 9 th phase.

The cycle selection step discarded an average of $3.25 \%$ of the counts. After the update of the average respiratory cycle duration, there were still $3.74 \%$ of omitted counts and $2.73 \%$ of twice used counts in a $4 \mathrm{D}$ reconstruction without cycle resampling. The trends in the repartition of the twice used counts among the phases were similar to those observed for G4D: e.g. an average of $26.86 \%$ (resp. $7.61 \%$ ) of counts used for phase 1 (resp. phase 2) were already used in a preceding phase.

After cycle resampling, all the retained counts are used once and only once in the $4 \mathrm{D}$ reconstruction G4DSR. Each reconstructed phase of G4DSR used around $6.45 \%$ of counts per reconstructed phase, which corresponded to a total of $96.75 \%$ counts over the 15 phases.

For the BH3D reconstruction, the counts from the equivalent of 9 or 10 phases have been used. On average $64 \%$ of the total number of counts have been used for the reconstruction of one $3 \mathrm{D}$ image.

\subsection{Qualitative comparison}

Each acquisition was reconstructed using the three methods NG3D, G4D and BH3D, that have been described in section 2 .

The first comparison was a visual comparison of the lesions in images reconstructed with the different methods. Figure 6 illustrates these reconstructions with sagittal views showing 2 peritoneal lesions. The peritoneal location can be visualised with the CT image (a). NG3D image (b) shows the underestimation of the lesion uptake and the overestimation of size compared to G4D images, with a significantly improved signalto-noise ratio. The zoomed-in images of the G4D end-of-exhalation (c) and end-ofinhalation $(\mathrm{d})$ images show the high lesion uptake and the respiratory induced lesion displacement, but are corrupted by a high noise level. On the BH3D reconstructed image (e), the obtained result is a trade-off between the previous methods: the outlines of the lesions are less blurred and the volumes are smaller than in NG3D image and closer to G4D end-of-exhalation image, while the lesion uptake is higher than in NG3D image with an equivalent noise level.

\subsection{Quantitative comparison}

NG3D, G4D and BH3D reconstruction methods were compared with respect to imagebased and lesion-based quantitative measurements. The average values of the lesions' $\mathrm{SUV}_{\text {peak }}, \mathrm{SUV}_{\text {mean }}$ and volumes, noise estimation in homogeneous liver area and signalto-noise ratio (SNR) are presented in table 3. Here, G4D images are reconstructed with both 6 phases and 15 phases. 
Image noise, measured in a homogeneous liver area, for BH3D method (see figure 7 (d)) was significantly reduced (-60.83\% and $-38.25 \%)$ in comparison to 15 phases-G4D and 6 phases-G4D $(p<0.001)$ and closer to NG3D measured noise, although it was still significantly higher (increase of $+21.37 \%, p=0.0012$ ).

The $\mathrm{SUV}_{\text {peak }}$ and $\mathrm{SUV}_{\text {mean }}$ for the BH3D method (see figure 7 (a)) show an increase of respectively $+9.67 \%$ and $+14.59 \%$ with respect to NG3D reconstruction $(p<0.001)$, but remained significantly lower (respectively $-5.29 \%$ and $-11.99 \%$ ) than those obtained by 15 phases-G4D reconstruction $(p<0.001)$. However, $\mathrm{SUV}_{\text {peak }}$ and $\mathrm{SUV}_{\text {mean }}$ were not significantly different to the ones for 6 phases-G4D reconstruction (slight decrease of respectively $-0.64 \%$ and $-1.2 \%)(p=0.9873$ and $p=0.5608)$. Volumes measured with BH3D method (see figure 7 (b)) were significantly lower $(-14.41 \%)$ than with NG3D $(p<0.001)$ and significantly higher $(+12.04 \%)$ than with G4D $(p<0.001)$, but were not significantly different $(-1.3 \%)$ to 6 phases-G4D $(p=0.655)$.

Lesion SNR on BH3D images was significantly higher $(+124 \%$ and $+55 \%)$ (see figure 7 (c)) than on both 15 phases- and 6 phases-G4D images $(p<0.001)$ and closer to NG3D lesion SNR, while it was still significantly smaller than on NG3D (-5.40\%) $(p=.0035)$.

\section{Discussion}

The motivation of this work is tumour detection and quantification in oncology small animal SPECT imaging. Respiratory motion is a major source of impairment not only for lung lesions but also for abdominal lesions as in our peritoneal carcinosis model. The gating reconstruction method was able to reconstruct motionless images but at the cost of a degraded SNR. Therefore we proposed a retrospective amplitude-based data selection that aimed to maximise the data available for reconstruction, hence keeping a high SNR, while suppressing respiratory motion artefacts.

The proposed method was possible thanks to the breathing rhythm particular to anaesthetised mice, which is characterised by gasping breaths separated by long intervals without respiratory motion. Cavanaugh et al. (Cavanaugh et al. 2004) previously reported that this pattern, which is also depicted in (Burk et al. 2012), was induced by isoflurane anaesthesia.

The average duration of the motionless baseline is 9.75 phases (for a 15-phases reconstruction), which corresponds to $65 \%$ of the respiratory cycle, which indicates that the same percentage of acquired counts may be used for the reconstruction of a motion free image.

We observed that the true motion, as observed in the CT or SPECT respiratory gated reconstruction (3D+t images), is not synchronised with the pressure signal, but appeared temporally shifted. This shift represents around a fourth of the cycle length (around $187 \mathrm{~ms}$ ). It has been identified as a consequence of the external pneumatic sensor system and might then depend on the length ot the air pipe. This asynchronism motivated a dedicated image-based motion detection method that allowed to identify 
the respiratory phases associated with the motionless baseline.

The proposed image-based motion detection method is simpler than those proposed in e.g. (Bundschuh et al. 2008) or (Schleyer et al. 2009). Indeed, both approaches aimed at reconstructing a respiratory representative signal from the images while we only focused on the detection of the phases exhibiting a motion with respect to baseline. More precisely, (Bundschuh et al. 2008) followed the centre of mass of a (manually specified) tumour in a $4 \mathrm{D}$ series and then used the measured displacement as a trigger signal. This approach required some manual interaction and is dedicated to the reconstruction of one single tumour. The proposed method is closer to (Schleyer et al. 2009) since we study the variation of counts induced by an object motion. They proposed to integrate this variation in the sinogram to get a respiratory-like signal while we simply identified the largest variations at the voxel level. As with theirs, our method is versatile and can deal either with emission tomography or CT data. The proposed method appears to be quite robust with respect to noise, and is still able to detect the motion in noisy challenging images (SNR of 2.5), noisier than the pre-clinical images (see the SNRs in Table 3).

The average time shift between the image-based detected motion and the amplitude peak in the pressure signal was -3.875 phases(for 15 phases-G4D), which corresponds to $25 \%$ of the respiratory cycle. Rather than using a 4D CT image for the motion detection, that would imply a large acquisition time and a subsequent radiation dose, we rely on a 4D SPECT image. Although the operator tried to stabilize the respiratory cycle of the anaesthetised mice, some variation still occurred (Table 1). As a consequence, some counts may be skipped from the reconstruction of gated images and others will be used twice, mostly in the first phases to be reconstructed. This motivated the equalization (done by resampling) of all respiratory cycles before reconstruction.

The proposed data selection allowed to retain $64 \%$ of all detected counts to reconstruct one single 3D image (BH3D). These counts correspond to the motionless baseline, hence the proposed reconstruction method simulated a breath-hold acquisition.

Using all acquired counts in the reconstruction would have required to either coregister all 3D phase image and then average them (e.g. (Dawood et al. 2006)), or to incorporate a motion model into the reconstruction process (e.g. (Reyes et al. 2007)) This would then either rely on some non-linear registration method, whose validation for such noisy data is challenging, or on the calculation of a personnalized respiratory motion model. 4D CT may offer a means for such computation, but will still depends on the used registration algorithm and at the cost of a high radiation dose of the mice. Therefore, the proposed method offers a means toward the reconstruction of a still image, while not requiring the validation of any registration method.

Measures of image noise (figure $7 \mathrm{~d}$ ) and lesion signal-to-noise ratio (figure $7 \mathrm{c}$ ) demonstrated that $\mathrm{BH} 3 \mathrm{D}$ is of better quality than a 3D phase image extracted from a 4D reconstruction (G4D, 6 and 15 phases reconstruction have been tested), and even suggested that it is of similar quality to the 3D image reconstructed with all the available counts (NG3D). Measures of $\mathrm{SUV}_{\text {mean }}$ are significantly larger in BH3D than in NG3D, 
and close to the ones from the phase image (G4D). Similarly, volume measures are smaller in BH3D than in NG3D, and close to G4D. These figures suggested that the blurring effect due to the respiratory motion has been compensated for. As expected, using less phases in G4D reconstruction yielded better quality images. The advantage of the proposed method is to discard prospectively the motion impaired data before reconstruction, while one has to identify retrospectively (possibly with a monitoring system) the motionless phases in G4D reconstruction. Last, the "optimal" G4D will reconstruct 2 phases, each of them from $50 \%$ of the data, assuming only one of them is impaired with motion, while our method used $64 \%$ of the data.

More thorough validation could be conducted with simulations. First, one would have to animate a numerical mouse phantom with the same particular respiratory pattern that has been observed (gasps followed by a rest period). Such a model has not been identified. The MOBY phantom (Segars et al. 2004) implemented a respiratory motion similar to humans, and would not be adequate. Moreover, we have no indication whether such a model may produce the same time shift as we observe between the pressure signal and the abdominal motion. Second the acquisition can be simulated (e.g. with the GATE software (Sakellios et al. 2006)), but this will also require to model our camera. Such a considerable amount of work is certainly worthwhile for a finer characterization of the benefits of the proposed method. However, the presented figures already suggest there is an the overall benefit.

\section{Conclusion}

We developed a reconstruction method dedicated to anaesthetised free-breathing mice. It enabled the reconstruction of a breath-hold like acquisition that is comparable to a non-gated reconstruction in terms of noise measure and signal-to-noise ratio, and intermediary between gated and non-gated reconstruction for lesion-based measurements ( $\mathrm{SUV}_{\text {peak }}, \mathrm{SUV}_{\text {mean }}$ and lesion volume).

Overall, the proposed method improves the quality of pre-clinical images, and the precision of the quantitative measurements they provide. This method is promising for more challenging studies concerning organs or tumour affected by motion using the emission tomography modalities in the pre-clinical research.

\section{Acknowledgments}

We thank Audrey Lamit for the supply of biological material, the planning of experimentation, the manipulation of animals, and the support during acquisition.

\section{References}

Bettinardi V, Picchio M, Di Muzio N, Gianolli L, Gilardi M \& Messa C 2010 Radiother Oncol 96(3), 3116.

Blondel C, Vaillant R, Malandain G \& Ayache N 2004 Phys Med Biol 49(11), 2197-2208. 
Bundschuh R, Martinez-Moller A, Essler M, Nekolla S, Ziegler S \& Schwaiger M 2008 Eur J Nucl Med Mol Imaging 35(11), 1981-8.

Burk L M, Lee Y Z, Wait J M, Lu J \& Zhou O Z 2012 Phys Med Biol 57(18), 5749-63.

Büther F, Ernst I, Dawood M, Kraxner P, Schäfers M, Schober O \& Schäfers K 2010 Eur J Nucl Med Mol Imaging 37(12), 2315-27.

Cavanaugh D, Johnson E, Price R, Kurie J, Travis E \& Cody D 2004 Mol Imaging 3(1), 55-62.

Chang G, Chang T, Pan T, Clark J \& Mawlawi O 2010 J Nucl Med 51(1), 16-24.

Dawood M, Lang N, Jiang X \& Schäfers K P 2006 IEEE Trans Med Imaging 25(4), 476-485.

Franc B, Acton P, Mari C \& Hasegawa B 2008 J Nucl Med 49(10), 1651-63.

Grotus N, Reader a J, Stute S, Rosenwald J C, Giraud P \& Buvat I 2009 Phys Med Biol 54(6), 1705-21.

Guo X, Johnston S, Qi Y, Johnson G \& Badea C 2011 Phys Med Biol 57(1), 257-271.

Hudson H M \& Larkin R S 1994 IEEE Trans Med Imaging 13(4), 601-609.

Hwang A, Franc B, Gullberg G \& Hasegawa B 2008 Phys Med Biol 53(9), 2233-52.

Kang J \& Chung J K 2008 J Nucl Med 49 Suppl 2, 164.

Kovalski G, Israel O, Keidar Z, Frenkel A, Sachs J \& Azhari H 2007 J Nucl Med 48(4), 630-636.

Kuntz J, Dinkel J, Zwick S, Bauerle T, Grasruck M, Kiessling F, Gupta R, Semmler W \& Bartling S 2010 Phys Med Biol 55(7), 2069-85.

Kyriakou E \& McKenzie D 2011 Phys Med Biol 56(10), 2999-3013.

Lamare F, Ledesma Carbayo M, Cresson T, Kontaxakis G, Santos A, Cheze Le Rest C, Reader A \& Visvikis D 2007 Phys Med Biol 52(17), 5187-204.

Livieratos L, Rajappan K, Stegger L, Schäfers K, Bailey D \& Camici P 2006 Eur J Nucl Med Mol Imaging 33(5), 584-8.

Loening A \& Gambhir S 2003 Mol Imaging 2(3), 131-7.

Lucignani G 2009 Eur J Nucl Med Mol Imaging 36(2), 315-9.

Martiniova L, Schimel D, Lai E, Limpuangthip A, Kvetnansky R \& Pacak K 2010 Methods 50(1), 20-5.

Nehmeh S \& Erdi Y 2008 Semin Nucl Med 38(3), 167-76.

Nehmeh S, Haj-Ali A, Qing C, Stearns C, Kalaigian H, Kohlmyer S, Schoder H, Ho A, Larson S \& Humm J 2011 Med Phys 38(1), 531-8.

Otsu N 1975 IEEE Trans Syst Man Cyb C(1), 62-66.

Pysz M, Gambhir S \& Willmann J 2010 Clin Radiol 65(7), 500-16.

Reyes M, Malandain G, Koulibaly P M, Gonzalez-Ballester M A \& Darcourt J 2007 Phys Med Biol 52(12), 3579-600.

Ritt P, Vija H, Hornegger J \& Kuwert T 2011 Eur J Nucl Med Mol Imaging 38 Suppl 1, S69-77.

Sakellios N, Rubio J, Karakatsanis N, Kontaxakis G, Loudos G, Santos A, Nikita K \& Majewski S 2006 in 'IEEE Nuclear Science Symposium Conference Record' pp. 2000-2003.

Schleyer P J, O'Doherty M J, Barrington S F \& Marsden P K 2009 Phys Med Biol 54(7), 1935-50.

Segars W, Tsui B, Frey E, Johnson G \& Berr S 2004 Mol Imaging 6(3), 149-59.

van Elmpt W, Hamill J, Jones J, De Ruysscher D, Lambin P \& Ollers M 2011 Eur J Nucl Med Mol Imaging 38(5), 843-855-855. 


\section{Tables and table captions}

Table 1. Respiratory signal analysis for G4D (15 phases) reconstructions.

\begin{tabular}{llll}
\hline Acquisition & $\begin{array}{l}\text { Cycle } \mu \pm \sigma \\
(\mathrm{ms})\end{array}$ & $\begin{array}{l}\text { Motion duration } \\
(\mathrm{ms} / \mathrm{nb} \text { phases })\end{array}$ & $\begin{array}{l}\text { Time shift } \\
(\mathrm{ms} / \mathrm{nb} \text { phases })\end{array}$ \\
\hline 0182 & $762.8 \pm 112.9$ & $255(5)$ & $153(-3)$ \\
0183 & $740.5 \pm 152.7$ & $294(6)$ & $147(-3)$ \\
0185 & $751.0 \pm 48.9$ & $250(5)$ & $200(-4)$ \\
0186 & $765.6 \pm 43.6$ & $255(5)$ & $204(-4)$ \\
0187 & $712.5 \pm 44.6$ & $240(5)$ & $192(-4)$ \\
0189 & $702.9 \pm 100.4$ & $282(6)$ & $188(-4)$ \\
0190 & $658.5 \pm 52.1$ & $220(5)$ & $220(-5)$ \\
0191 & $713.8 \pm 59.1$ & $240(5)$ & $192(-4)$
\end{tabular}

Table 2. Percentages of counts used for the different SPECT images reconstruction schemes: NG3D, G4D (15 phases) and BH3D. Values are presented as mean +/standard deviation.

\begin{tabular}{llc}
\hline & \multicolumn{2}{c}{ Number of counts used (\%) } \\
\hline NG3D & Total & 100.00 \\
\hline G4D & Used counts / phase & $6.65 \pm 0.19$ \\
& Total & $99.76 \pm 2.88$ \\
& Omitted counts & $3.64 \pm 3.14$ \\
& counts used twice & $3.51 \pm 1.55$ \\
\cline { 2 - 2 } & Percentages of counts used twice per phase & $31.44 \pm 7.11$ \\
& phase 1 & $9.67 \pm 7.94$ \\
& phase 2 & $5.29 \pm 7.02$ \\
& phase 3 & $3.60 \pm 4.87$ \\
& phase 4 & $1.79 \pm 2.48$ \\
& phase 5 & $0.46 \pm 0.68$ \\
& phase 6 & $0.22 \pm 0.47$ \\
& phase 7 & phase 8 \\
& phase 9 & $0.10 \pm 0.26$ \\
& phases 10 to 15 & $0.01 \pm 0.04$ \\
\hline G4DSR & Used counts / phase & $6.45 \pm 0.29$ \\
& Total & $96.75 \pm 4.36$ \\
\hline BH3D & Total & $63.99 \pm 4.58$ \\
\hline
\end{tabular}


Table 3. Quantitative results for NG3D, G4D and BH3D reconstruction methods: $\mathrm{SUV}_{\text {peak }}, \mathrm{SUV}_{\text {mean }}$, lesion volume (threshold at $40 \%$ of the $\mathrm{SUV}_{\max }$ value), noise estimation in homogeneous liver area and signal-to-noise ratio (SNR). Values are presented as mean $+/$ - standard deviation.

\begin{tabular}{lrccccc}
\hline Parameters & \multicolumn{1}{c}{ NG3D } & & G4D $(15 \text { phases })^{\mathrm{a}}$ & G4D $(6 \text { phases })^{\mathrm{a}}$ & \multicolumn{1}{l}{ BH3D } \\
\hline Noise $^{\mathrm{b}}$ & $0.15 \pm$ & 0.04 & $0.47 \pm 0.14$ & $0.30 \pm 0.08$ & $0.18 \pm 0.05$ \\
SUV $_{\text {peak }}$ & $7.26 \pm 5.32$ & $8.18 \pm 5.56$ & $7.87 \pm 5.46$ & $7.87 \pm 5.47$ \\
SUV $_{\text {mean }}$ & $6.90 \pm$ & 5.26 & $8.63 \pm 6.00$ & $7.85 \pm 5.67$ & $7.80 \pm 5.65$ \\
Volume $\left(\mathrm{mm}^{3}\right)$ & $2.14 \pm$ & 1.29 & $1.60 \pm 0.87$ & $1.84 \pm 1.00$ & $1.79 \pm 1.02$ \\
${\text { SNR }\left(\mathrm{SUV}_{\text {mean }} / \text { Noise }\right)}$ & $48.85 \pm 40.02$ & $19.81 \pm 14.98$ & $31.82 \pm 22.50$ & $45.61 \pm 35.86$
\end{tabular}

${ }^{a}$ G4D values are the average values of end-of-exhalation images corresponding to the motionless phases - i.e. 9 or 10 phases (resp. 4) in the case of G4D with 15 phases (resp. 6 phases).

b Noise measure refers to the standard deviation in a homogeneous liver area. 


\section{Figure captions}

Figure 1. Histogram of cycle duration This figure presents the distribution of cycle duration for a pressure signal record, with its Gaussian curve fit. Vertical red dotted lines indicate the average cycle duration $\bar{C}$ (middle) and the two limits $\bar{C}-3 \sigma$ and $\bar{C}+3 \sigma$ that have been defined for the cycle selection. Cycle with a duration outside the limits are discarded for following reconstruction.

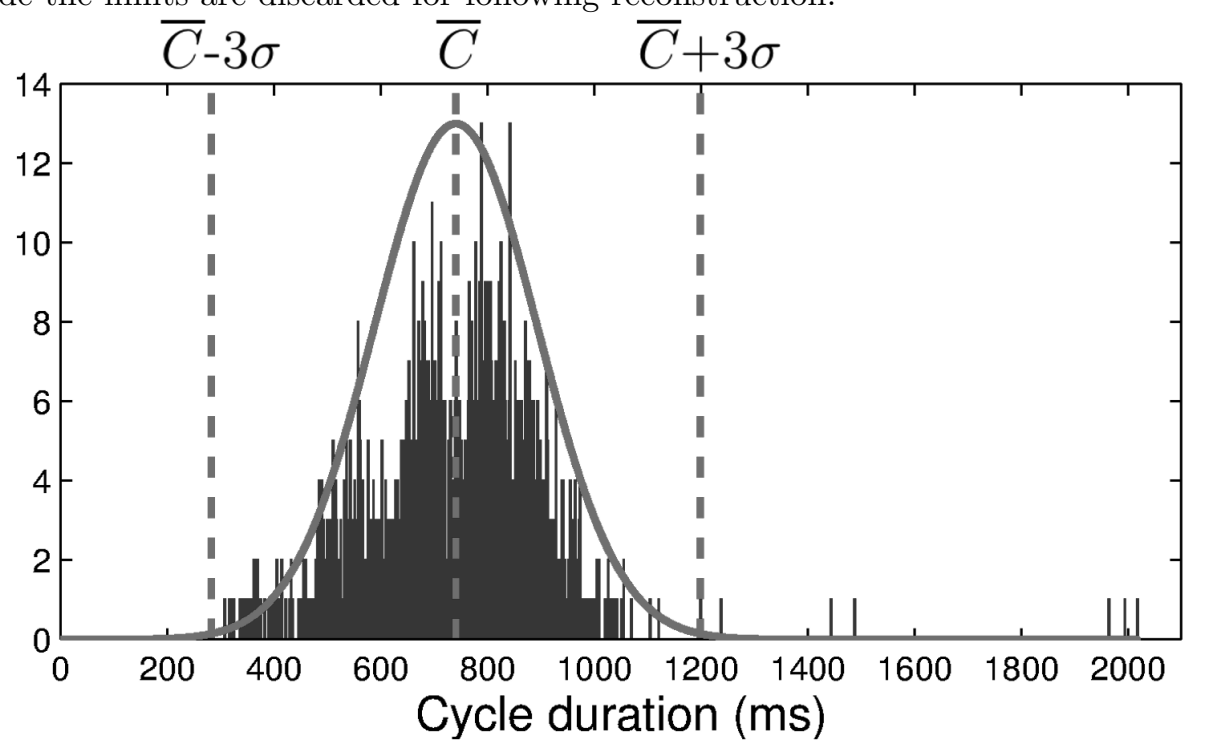


Figure 2. Reconstruction windows for G4D reconstruction The two graphs represent two samples of the same pressure signal. Vertical lines delimit the reconstruction windows for each phase of the G4D image. Sample of pressure signal with cycles shorter than the average duration $\bar{C}_{f}$ highlights the overlapping of phases from consecutive cycles (a); Sample of pressure signal with cycles longer than $\bar{C}_{f}$ highlights the fact that data at the end of some cycle are not used for reconstruction (b). Moreover, to reconstruct a phase of the G4D image (for example phase 15), G4D reconstruction use data that does not correspond to the same time in the respiratory cycle. Sometimes it corresponds to the end of exhalation (a), sometimes it corresponds to the end of inhalation (b).

(a)

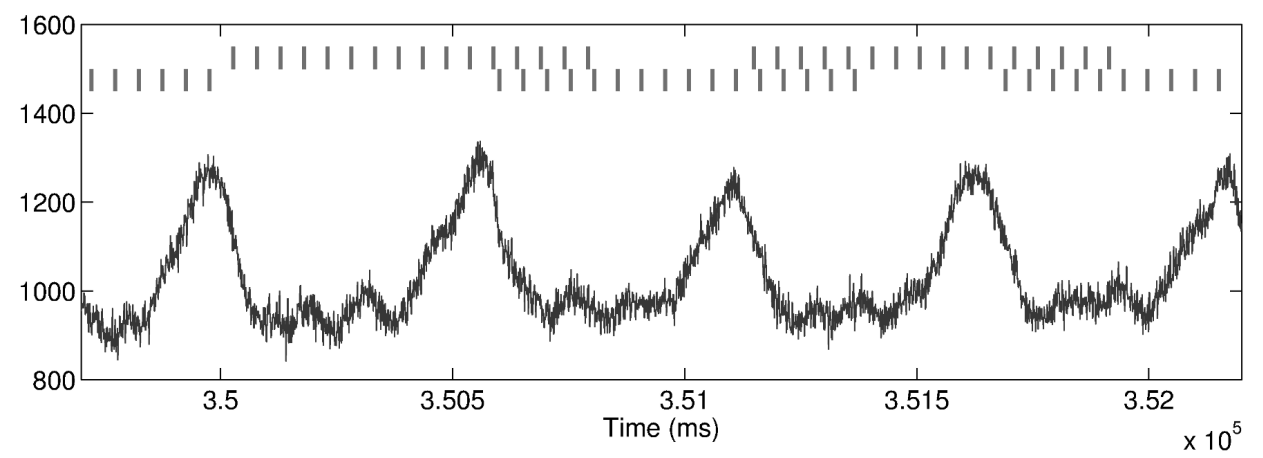

(b)

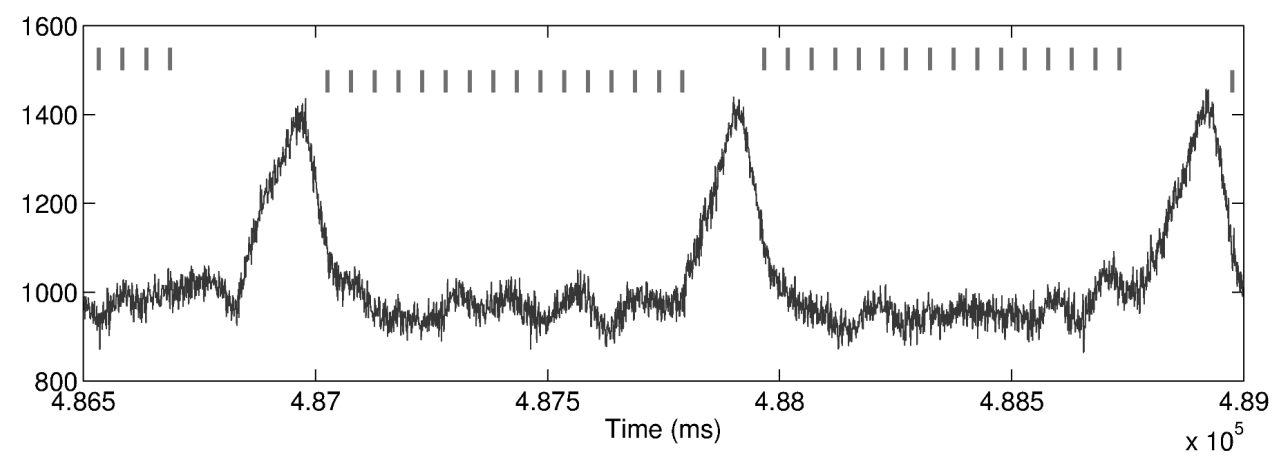


Figure 3. Singularity of anaesthetised mice pressure signal with motion phase detection Pressure signal recorded with the monitoring system (a); and normalised average pressure signal (continuous curve) and normalised histogram resulting from the image-based motion phase detection (dashed curve), the horizontal line depicts the threshold determined by the Otsu method (b). The phases getting more votes (above the threshold) are associated with the motion phase and the ones with less votes (below the threshold) are associated with the motionless phase.
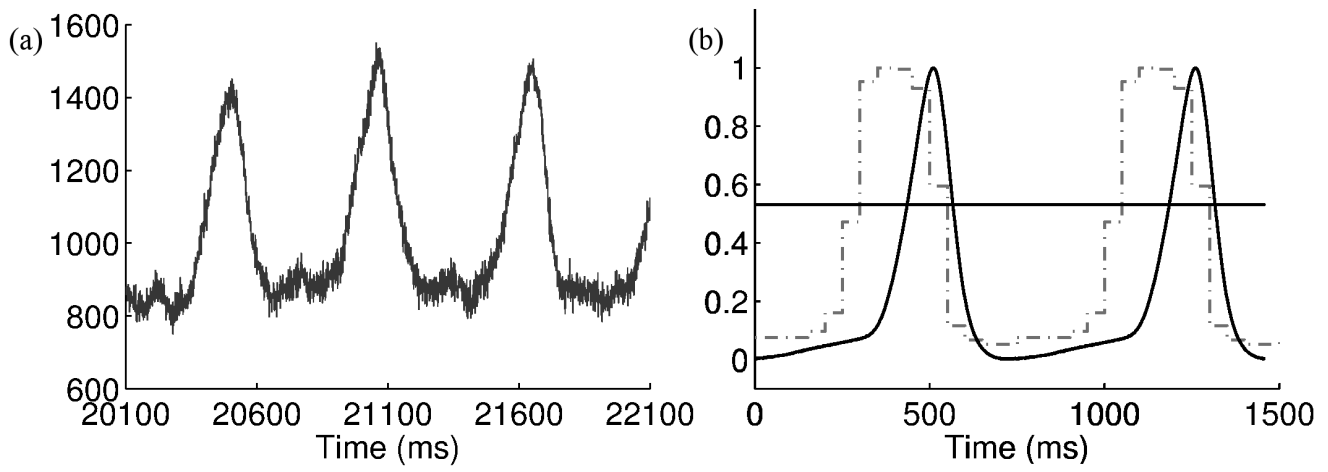
Figure 4. CT/SPECT volume rendering highlighting peritoneal carcinoma lesions. Volume rendering of fused CT and SPECT (NG3D) highlighting peritoneal carcinoma lesions after 3 weeks of growth, salivary glands and stomach (S).

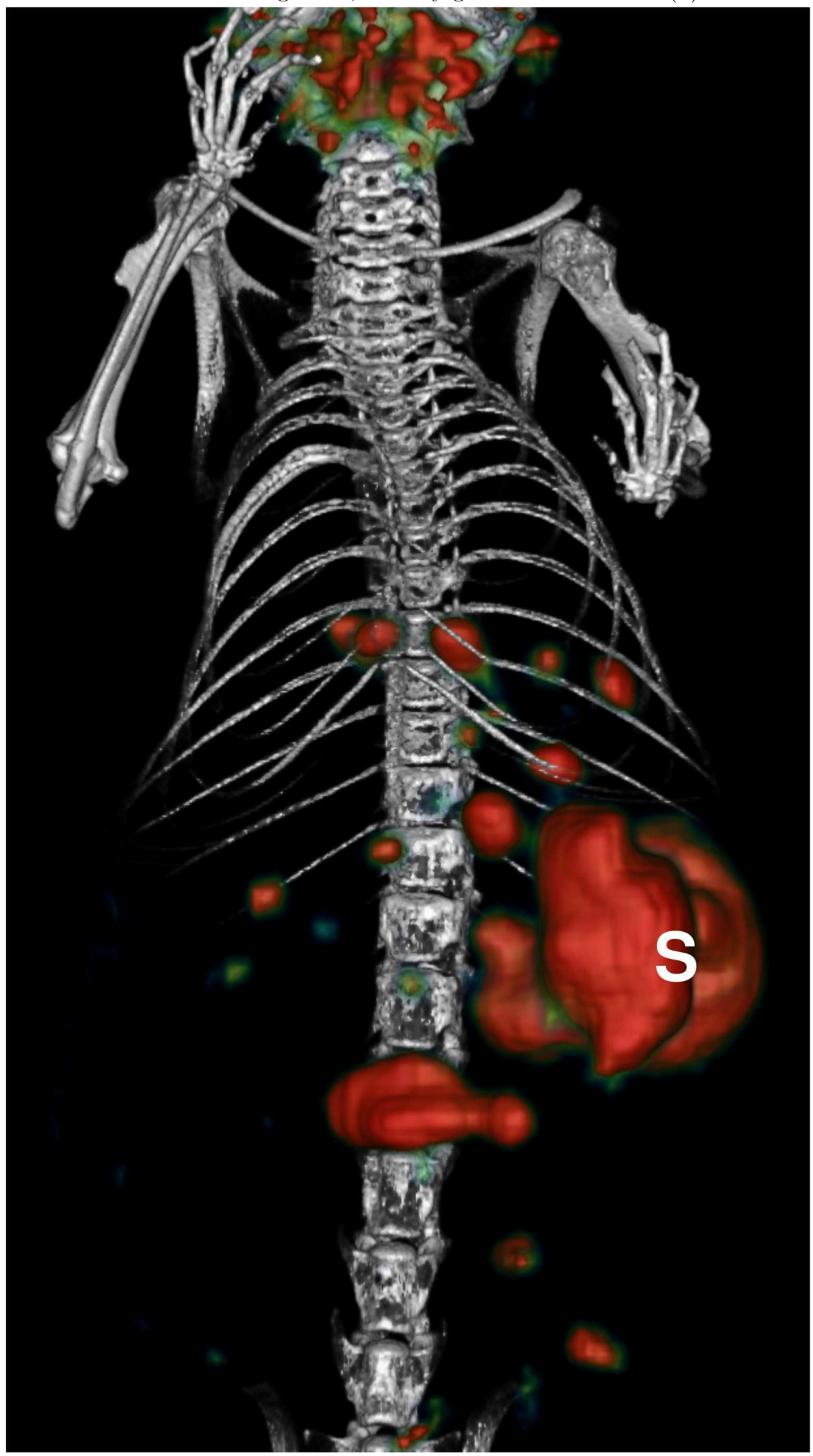


Figure 5. Comparison between pressure signal and image-based measures computed on 4D-SPECT and 4D-CT. Normalised average pressure signal (continuous curve); normalised histogram $h_{4 D-S P E C T}(n)$ computed on the $4 \mathrm{D}-\mathrm{SPECT}$ image (staircase); normalised histogram $h_{4 D-C T}(n)$ of motion phase computed on the 4D-CT image (gray curve with cross-shaped markers); and lung volume $(\mathrm{mL})$ measured on the 4D-CT image (dotted curve with circle-shaped markers).

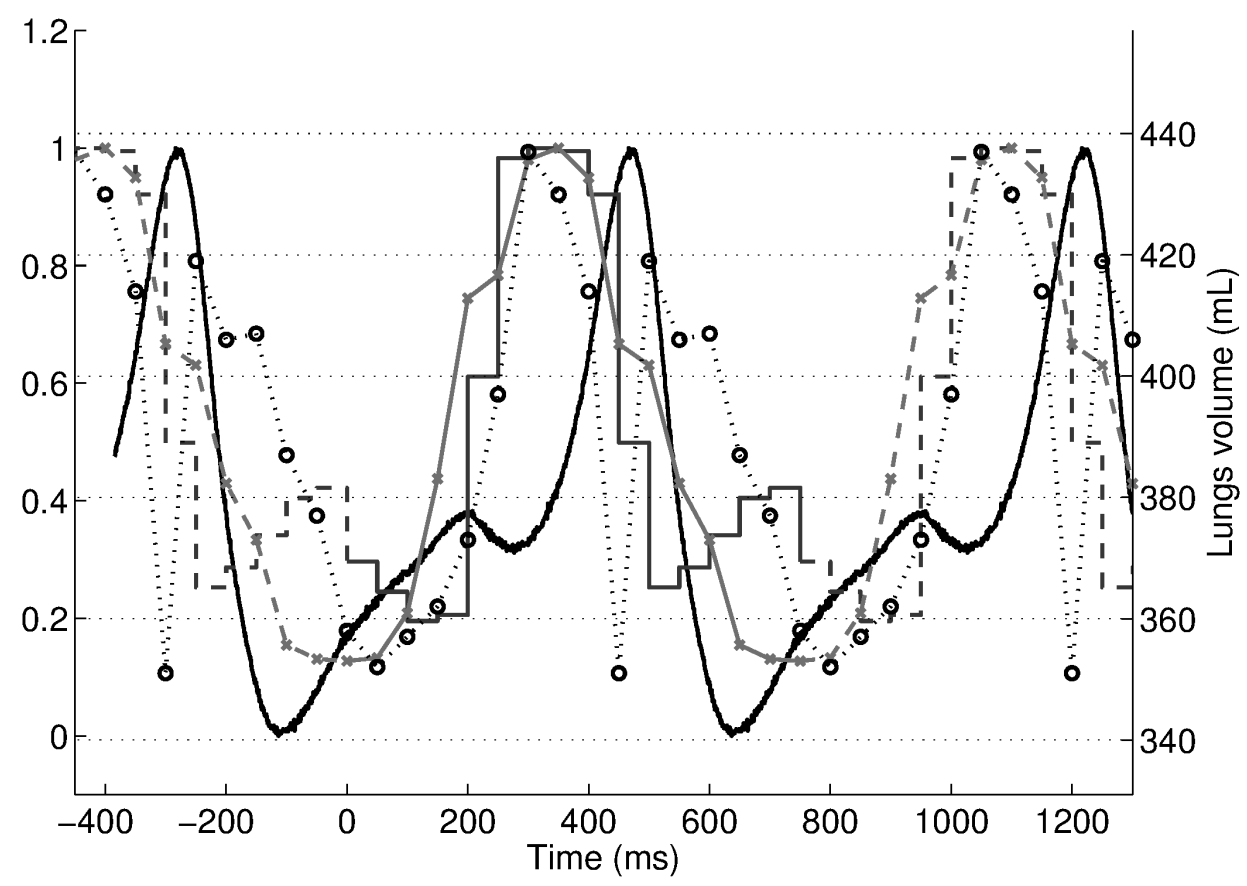


Figure 6. Sagittal views from sub-diaphragmatic peritoneal lesions comparing NG3D, G4D (15 phases), BH3D SPECT reconstruction methods. Top row: anatomical reference CT image (a), NG3D reconstruction (b), end-of-exhalation phase from G4D reconstruction (c), end-of-inhalation phase from G4D reconstruction (d), BH3D reconstruction (e); bottom row: zoomed-in on lesions of interest of the SPECT images. The SUV colour map has been set up on $\mathrm{SUV}_{\max }$ values in G4D images for the selected lesion.

(a)

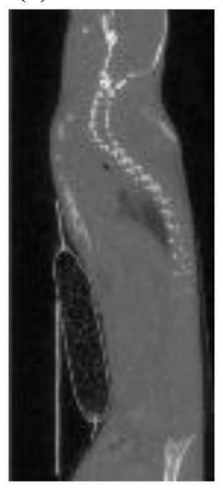

(b)

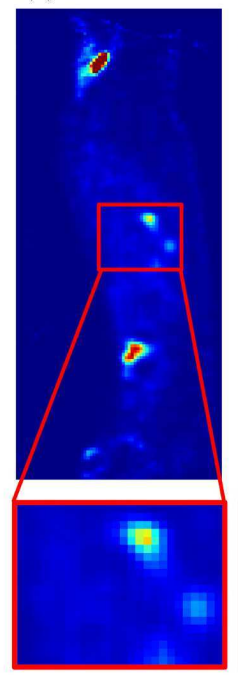

(c)

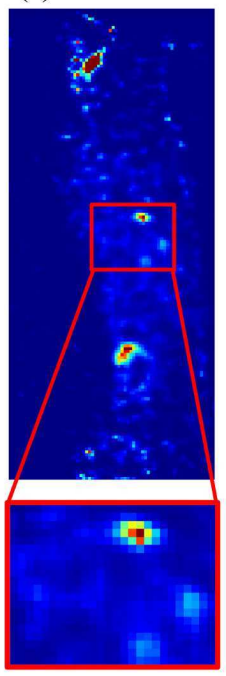

(d)

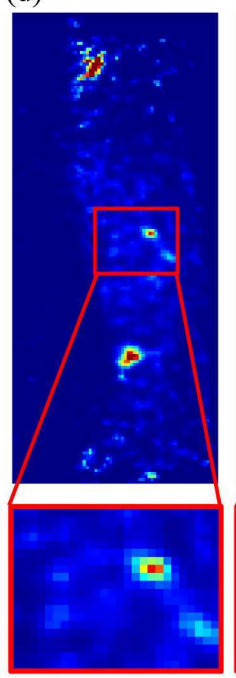

(e)

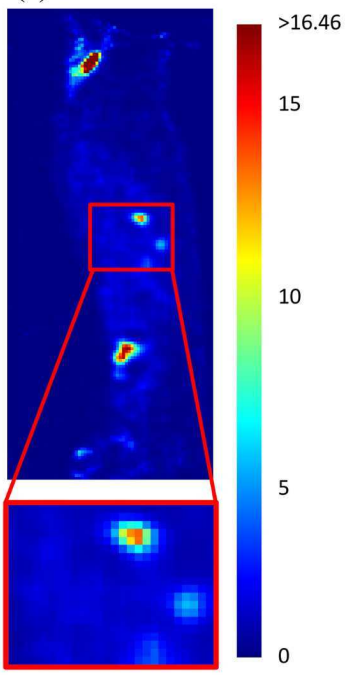


Figure 7. Quantitative results for lesions and image characteristics Quantitative results for lesions $\mathrm{SUV}_{\text {mean }}(\mathbf{a})$, lesions volumes (b), signal-to-noise ratio (SNR) (c), and noise (d). Box plots show the median, upper and lower quartiles and range; + indicate outliers. Statistical significance: ** for $p<0.01,{ }^{* * *}$ for $p<0.001$, $p$-value is given when non significant $(p>0.05)$.
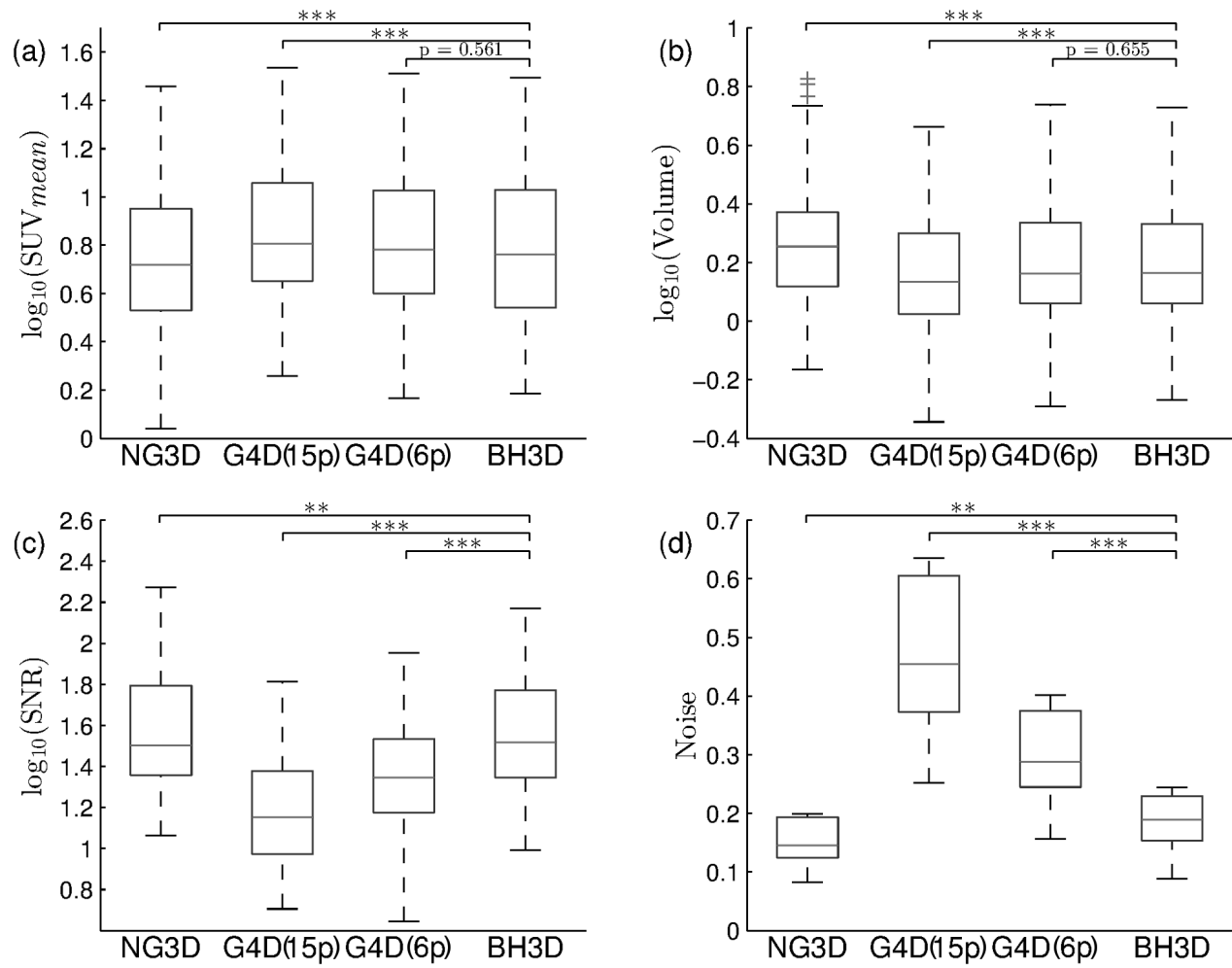